\title{
Pérdidas de Glifosato por Drenaje y Escurrimiento en Molisoles bajo Siembra Directa
}

\author{
María C. Sasal ${ }^{(1)}$, Adrian E. Andriulo(2), Marcelo G. Wilson ${ }^{(1)}$, Silvina I. Portela ${ }^{(2)}$ \\ Instituto Nacional de Tecnología Agropecuaria, (1) EEA Paraná, Ruta 11, km 12,5, Oro Verde, \\ Entre Ríos, Argentina, (2) INTA EEA Pergamino, Ruta 32, Km 5,5, Pergamino, Buenos Aires- \\ Argentina (e-mail: csasal@parana.inta.gov.ar, andriulo@pergamino.inta.gov.ar, \\ mwilson@parana.inta.gov.ar, sportela@pergamino.inta.gov.ar)
}

Recibido Oct. 26, 2009; Aceptado Nov. 26, 2009; Versión Final recibida Ene. 07, 2009

\begin{abstract}
Resumen
Se ha cuantificado las pérdidas de glifosato por drenaje y escurrimiento y su translocación hacia el grano en soja transgénica sembrada en Molisoles representativos de la Pampa Ondulada argentina bajo siembra directa. Se utilizaron 3 lisímetros (Argiudol típico) y 3 parcelas de escorrentía (Argiudol ácuico) ubicados en las Estaciones Experimentales del Instituto Nacional de Tecnología Agropecuaria de Pergamino y Paraná, respectivamente. El glifosato aplicado antes de la siembra se detectó en el agua de drenaje mientras que la aplicación de post-emergencia fue detectada en el grano. Se registraron máximos de elevadas concentraciones de glifosato en el agua de drenaje y escurrimiento $\left(\sim 10 \mu \mathrm{g} \mathrm{L}^{-1}\right)$. Sin embargo, la cantidad de glifosato que salió del sistema representó menos del 0,03 y $0,6 \%$ de las cantidades aplicadas, respectivamente. Se observó elevada variabilidad de las concentraciones de glifosato en todos los compartimentos analizados.
\end{abstract}

Palabras claves: glifosato, drenaje, escurrimiento, soja transgénica, Molisoles

\section{Glyphosate losses by drainage and runoff from Mollisols under no-till agriculture}

\begin{abstract}
Glyphosate losses by drainage and runoff from no-till-soybean-cultivated Molisolls representative of the Argentinean Rolling Pampas and its translocation in transgenic soybean biomass have been determined. Three lisymeters (Typic Argiudoll) and 3 runoff plots (Aquic Argiudoll) located in the Experimental Stations of the National Institute of Farming Technology of Pergamino y Paraná, respectively, were used. Glyphosate applied before sowing was detected in drainage water and the post-emergence application was detected in grains with small and variable concentrations. Peaks of glyphosate concentrations in drainage and runoff water were registered after important rain events $\left(\sim 10 \mu \mathrm{L} \mathrm{L}^{-1}\right)$. However, the amount of glyphosate lost throughout the study period was lower than 0.03 and $0.6 \%$ of the amounts applied, respectively. High variability of Glyphosate concentrations was found in all compartments analyzed.
\end{abstract}

Keywords: glyphosate, drainage, runoff, transgenic soybean, Mollisols 


\section{INTRODUCCIÓN}

Existe creciente preocupación en la sociedad por la conservación de los recursos naturales y, en los últimos años, la agricultura está señalada como responsable de su degradación. En consecuencia, debe atenderse la demanda social del cuidado de la salud y del ambiente con datos fehacientes del impacto de la agricultura sobre la calidad del agua y los alimentos.

En la actualidad el glifosato ( $\mathrm{N}$-fosfonometil glicina) es el herbicida más utilizado a escala mundial para el control de malezas en áreas agrícolas y urbanas (Borggaard, Gimsing 2008). Es un herbicida organofosforado de acción sistémica postemergente que aplicado sobre el follaje es asimilado por las hojas y rápidamente translocado (Sprankle et al., 1975). Su acción no selectiva y de amplio espectro, se basa en la inhibición de la síntesis de aminoácidos esenciales. En el suelo es adsorbido mediante uniones fosfato y degradado por microorganismos. Esto determina una escasa movilidad tanto del glifosato como de su principal metabolito, el ácido amino metil fosfónico (AMPA). Sin embargo, estudios de lixiviación y escurrimiento indican que el glifosato puede ser transportado hacia las capas profundas del suelo debido al flujo preferencial por macroporos o hacia cursos de agua superficiales cuando su aplicación se realiza en momentos anteriores a lluvias intensas (Pertensen et al., 2002; Jaynes et al., 2001; Elliot et al., 2000; Villholth et al., 2000 y Flury 1996).

Si bien la concentración de sustancias disueltas en el drenaje profundo o en el escurrimiento superficial se diluye cuando estos descargan en los cursos de agua, se admite como nivel umbral que las concentraciones de plaguicidas que salen de un sistema agrícola no superen los límites admitidos para el consumo humano. En Europa este límite es de $0,1 \mathrm{\mu g}^{-1}$ para glifosato y AMPA (Vereecken, 2005). En USA, este nivel es más elevado, aunque hay importantes diferencias (entre 6 y $700 \mathrm{mg} \mathrm{I}^{-1}$ ) entre estados (USEPA, 2002). Byer et al. (2008) analizaron 739 muestras de agua superficial en 150 sitios de Ontario (Canadá) y detectaron glifosato en el 33\% de las muestras con concentraciones máximas de $12 \mu \mathrm{g} \mathrm{I}^{-1}$.

La agricultura argentina se está tornando cada vez más especializada y homogénea. En efecto, el esquema de producción a partir de la década del 90 se caracteriza por grandes superficies bajo siembra directa continua (SD) y alta proporción del cultivo de soja. En el país, la superficie destinada a este cultivo se ha casi duplicado en los últimos 10 años llegando a los niveles récord de producción de 47,5 millones de toneladas en la campaña 2007/2008 (SAGPYA, 2008). El uso casi exclusivo de variedades transgénicas de soja está ligado al uso del herbicida glifosato. Esto justifica la necesidad de evaluar el impacto ambiental que genera su uso en esta región. Peruzzo et al. (2008) registraron recientemente concentraciones de glifosato de 0,10 a $0,70 \mathrm{mg} \mathrm{I}^{-1}$ en 4 cursos de agua del área agrícola de la Pampa Ondulada argentina y Arregui et al. (2004) lo detectaron en tejidos y granos de soja transgénica de la misma región.

Aunque se ha avanzado mucho en el estudio de la acción del glifosato sobre las malezas y su inocuidad para el cultivo transgénico, la comprensión de sus vías de degradación ambientales (persistencia y destino después de cumplir con su efecto fitotóxico) permanece incompleta. En efecto, existe poca información sobre las pérdidas de glifosato a través de los excedentes hídricos y más aún sobre su acumulación en grano. El objetivo de este trabajo fue cuantificar las pérdidas de glifosato por drenaje profundo y escurrimiento y su translocación hacia el grano, en soja transgénica sembrada en Molisoles representativos de la Pampa Ondulada bajo siembra directa. Cuantificar estas pérdidas y conocer su dinámica aportaría información sobre aspectos relacionados con el mejor momento de su aplicación para tener un menor impacto sobre el ambiente.

\section{MATERIALES Y MÉTODOS}

El estudio fue realizado en dos Estaciones Experimentales del Instituto Nacional de Tecnología Agropecuaria (INTA) ubicadas en la Pampa Ondulada (Argentina): Pergamino en la provincia de

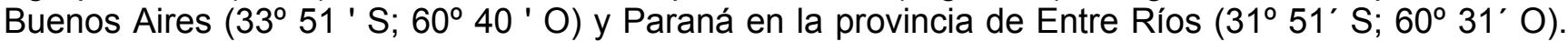
La región tiene clima subhúmedo (precipitación anual $\approx 1000 \mathrm{~mm}$ ) y templado $\left(17,0\right.$ y $18,3^{\circ} \mathrm{C}$ de temperatura media anual para Pergamino y Paraná, respectivamente). El $75 \%$ de las lluvias anuales ocurren en primavera y verano. En general, las temperaturas invernales son superiores a $0{ }^{\circ} \mathrm{C}$ y los veranos son muy cálidos. Entre el 50 y el $75 \%$ de la tierra se destina a la producción de granos (Hall 
et al 1992) y el principal cultivo es la soja. En el área predominan los suelos Molisoles de textura franco limosa en su horizonte superficial con $20-30 \%$ y $60-65 \%$ de arcilla y limo, respectivamente. Debajo del horizonte superficial yace un horizonte argílico que generalmente limita la permeabilidad del agua y la penetración de raíces.

Para medir drenaje se utilizaron tres cajas lisimétricas de hormigón armado (lisímetros cerrados y de gravedad) ubicadas en la Estación Experimental de Pergamino. Cada lisímetro tiene una superficie de $10 \mathrm{~m}^{2}(3,3 \times 3,3 \times 1,4 \mathrm{~m}$ de profundidad) y no presentan pendiente. Las cajas lisimétricas fueron construídas en 1969 y rellenadas con un Argiudol típico de la serie Pergamino (Luvic Phaeozem, WRB), respetando la secuencia, el espesor y la densidad aparente de cada uno de los horizontes del perfil (Tabla 1). Los perfiles se apoyan sobre un lecho filtrante de arena y grava que deriva el agua hacia un tubo de salida para recoger el drenaje a la profundidad de 1,4 $\mathrm{m}$ (Figura 1). Este dispositivo ha sido utilizado durante 30 años con diferentes cultivos: maíz, trigo, soja y girasol con riego complementario. En 2001 se inició una rotación maíz-soja bajo SD.

Tabla 1. Características físicas y químicas medias del suelo contenido en los lisímetros: Argiudol típico (Serie Pergamino). En la Tabla, CC y PMP son la capacidad de campo y el punto de marchitéz permanente

\begin{tabular}{|c|c|c|c|c|c|}
\hline \multicolumn{2}{|l|}{ Horizonte } & \multicolumn{2}{|c|}{$A+B A$} & \multirow{2}{*}{$\frac{B t}{0,30-1,00}$} & \multirow{2}{*}{$\frac{B C}{1,00-1,20}$} \\
\hline Espesor & $\mathrm{m}$ & $0-0,05$ & $0,05-0,30$ & & \\
\hline Arcilla & \multirow{3}{*}{$\%(w / w)$} & 21,6 & 20,6 & 38,8 & 19,0 \\
\hline Limo & & 58,4 & 55,6 & 43,5 & 55,9 \\
\hline Arena & & 20,0 & 23,8 & 17,7 & 25,1 \\
\hline Textura & & \multicolumn{2}{|c|}{ Franco limoso } & $\begin{array}{l}\text { Franco arcillo } \\
\text { limoso }\end{array}$ & Franco limoso \\
\hline $\begin{array}{l}\text { Densidad } \\
\text { aparente }\end{array}$ & $\mathrm{g} \mathrm{cm}^{-3}$ & 1,10 & 1,30 & 1,35 & 1,25 \\
\hline$C C$ & \multirow{2}{*}{$\%(w / w)$} & 28,9 & 27,0 & 27,6 & 25,4 \\
\hline$P M P$ & & 14,6 & 15,4 & 18,5 & 13,9 \\
\hline $\mathrm{pH}_{\text {agua }}$ & $(1: 2.5)$ & 6,2 & 6,6 & 6,9 & 7,6 \\
\hline C orgánico & $\mathrm{g} \mathrm{kg}^{-1}$ & 24,7 & 16,1 & 5,5 & 4,3 \\
\hline
\end{tabular}

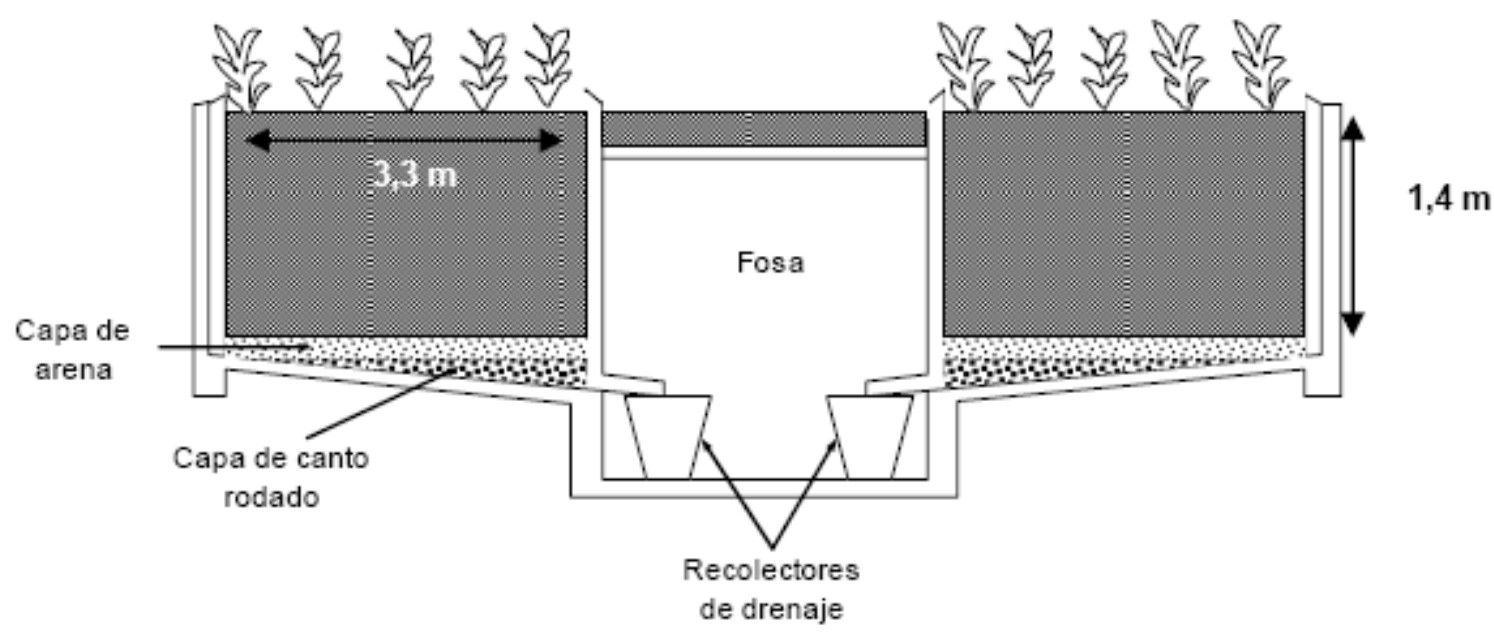

Fig. 1. Esquema de los lisímetros (corte transversal sin escalar) utilizados para medir el drenaje y su concentración de glifosato. 
Para medir escurrimiento se utilizaron tres parcelas de $100 \mathrm{~m}^{2}(4 \times 25 \mathrm{~m}$ y $3,5 \%$ de pendiente), construidas en la Estación Experimental Paraná en 1970 (Figura 2), sobre un Argiudol ácuico de la serie Tezanos Pinto (Tabla 2). Estas parcelas fueron diseñadas para medir los coeficientes de cultivos para aplicar en la ecuación universal de pérdida de suelo (Convenio FAO-INTA, 1969-1974). Desde 1990 se cultivan bajo SD, para adecuar la información al sistema adoptado por la mayor parte de los productores que trabajan en zonas onduladas.
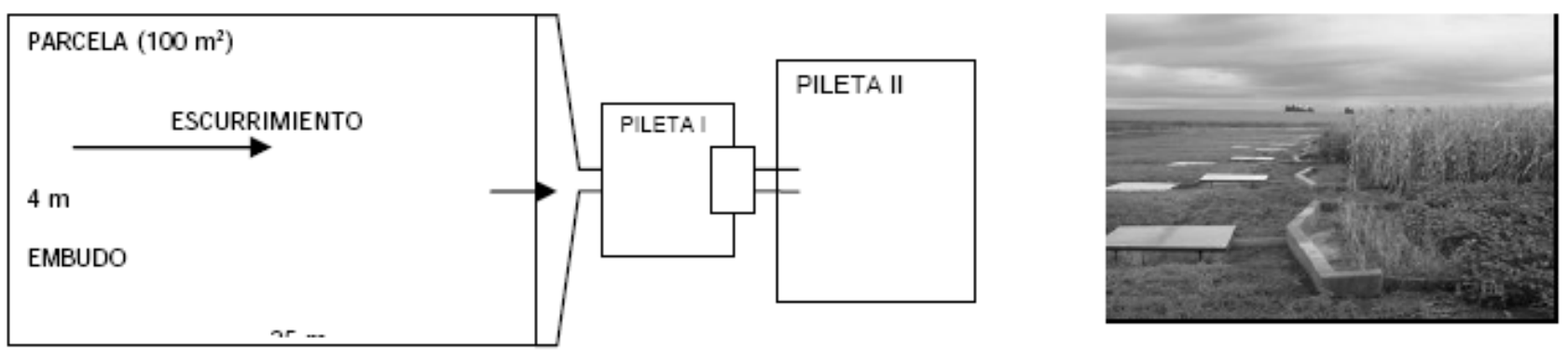

Fig. 2: Esquema (vista superior sin escalar) del dispositivo utilizado para medir escurrimientos y pérdidas de glifosato.

Tabla 2: Características físicas y químicas medias del horizonte $A$ de las parcelas de escurrimiento: Argiudol ácuico (Serie Tezanos Pinto). (CC y PMP: capacidad de campo y punto de marchitéz permanente, respectivamente. Dap: Densidad aparente).

\begin{tabular}{|c|c|c|c|c|c|c|c|c|c|}
\hline Horizonte A & Arcilla & Limo & Arena & Textura & $C C$ & PMP & Dap & C total & $\begin{array}{c}\text { Macroporosidad } \\
(>50 \mu m)\end{array}$ \\
\hline$m$ & & $\%$ & & & \multicolumn{2}{|c|}{$\%(w / w)$} & g. $\mathrm{cm}^{-3}$ & $g \mathrm{~kg}^{-1}$ & $\%$ \\
\hline $0-0,05$ & \multirow{2}{*}{26,0} & \multirow{2}{*}{66,0} & \multirow{2}{*}{8,0} & \multirow{2}{*}{$\begin{array}{l}\text { Franco } \\
\text { limoso }\end{array}$} & \multirow{2}{*}{25,1} & \multirow{2}{*}{16,5} & 1,23 & 26,9 & 9,55 \\
\hline $0,05-0,17$ & & & & & & & 1,34 & 17,1 & 5,4 \\
\hline
\end{tabular}

En ambos dispositivos se sembró soja (Glycine max (L) Merr.) transgénica en el mes de noviembre. En Pergamino se realizó una aplicación de glifosato un mes antes de la siembra (7-10-02) y otra en post-emergencia (28-11-02). En Paraná se realizó la primera aplicación de glifosato en presiembra $(27 / 09 / 06)$ y luego dos más en pre $(14 / 11 / 06)$ y post-emergencia $(11 / 12 / 06)$. Todas las aplicaciones tuvieron una dosis de $3 \mathrm{I}$ ha $^{-1}$ de glifosato (p.a. 48\%) sal de isopropilamina.

Se analizaron datos de las campañas 2002/2003 en Pergamino y 2006/2007 en Paraná, que tuvieron precipitaciones anuales de 1433 y $1574 \mathrm{~mm}$, respectivamente ( $50 \%$ superiores al promedio histórico en ambos casos). El registro de las precipitaciones se realizó en los Observatorios Agrometeorológicos de las respectivas Estaciones Experimentales, situados a $50 \mathrm{~m}$ y $200 \mathrm{~m}$ de los dispositivos para Pergamino y Paraná, respectivamente. Después de cada lluvia que produjo drenaje o escurrimiento se midió su volumen y se extrajo una muestra para determinar la concentración de glifosato y su metabolito AMPA. En Pergamino se registró un evento de drenaje antes de la primera aplicación, 5 entre la primera y segunda aplicación y 5 entre la segunda aplicación y la cosecha. En Paraná se agruparon las muestras de agua de escurrimiento y se analizaron glifosato y AMPA en 7 momentos del ciclo del cultivo. A la cosecha se analizó glifosato en grano sólo en Pergamino. Para ello, se extrajeron muestras de tallos, vainas y granos en cada lisímetro.

Las muestras de agua y tejidos vegetales se analizaron con HPLC con derivatización post-columna y detección por fluorescencia (límite de detección: $0,05 \mathrm{\mu g} \mathrm{I}^{-1}$ en agua y $0,02 \mathrm{mg} \mathrm{kg}^{-1}$ en grano). Las determinaciones se realizaron en el Instituto de Desarrollo Tecnológico para la Industria Química (CONICET-UNL). La cantidad de glifosato en agua de drenaje y/o escurrimiento se calculó como el producto del volumen drenado o escurrido por la concentración medida en el agua de drenaje o escurrimiento del mismo evento y se expresó en $\mu \mathrm{g} \mathrm{ha}^{-1}$. 


\section{RESULTADOS Y DISCUSIÓN}

En Pergamino, se detectó glifosato en el agua de drenaje en tres fechas de muestreo con

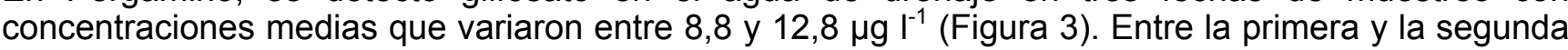
aplicación de glifosato se registraron $340 \mathrm{~mm}$ de lluvia, de los cuales 90 se perdieron por drenaje profundo. La presencia de vías de flujo preferencial y en particular de macroporos, común en suelos muy estructurados y conducidos bajo SD, favoreció la lixiviación de glifosato. Entre la segunda aplicación de glifosato y la cosecha se registraron $688 \mathrm{~mm}$ de lluvia y $60 \mathrm{~mm}$ de drenaje, pero no se detectó glifosato en el agua de drenaje. Esto se atribuyó a la intercepción del glifosato aplicado en post-emergencia del cultivo de soja.

La cantidad de glifosato lixiviada representó entre 0,3 y $0,6 \%$ del aplicado. Las elevadas precipitaciones del período evaluado también produjeron pérdidas de agua por escurrimiento estimadas en $80 \mathrm{~mm}$ para condiciones de cultivo y suelo similares a las de los lisímetros (Portela et al., 2009). El $70 \%$ de estas pérdidas se concentraron en el mes de octubre (presiembra) y seguramente contribuyeron a incrementar las fugas de glifosato hacia arroyos. De modo que las cantidades de glifosato lixiviadas podrían estar sobreestimadas por el aumento en el volumen de agua drenado en ausencia de escurrimiento superficial en los lisímetros.

En general, en todos los momentos de muestreo la variabilidad de las concentraciones de glifosato resultó muy elevada y estuvo condicionada por la imposibilidad de aumentar el número de repeticiones debido a las características de las unidades experimentales.

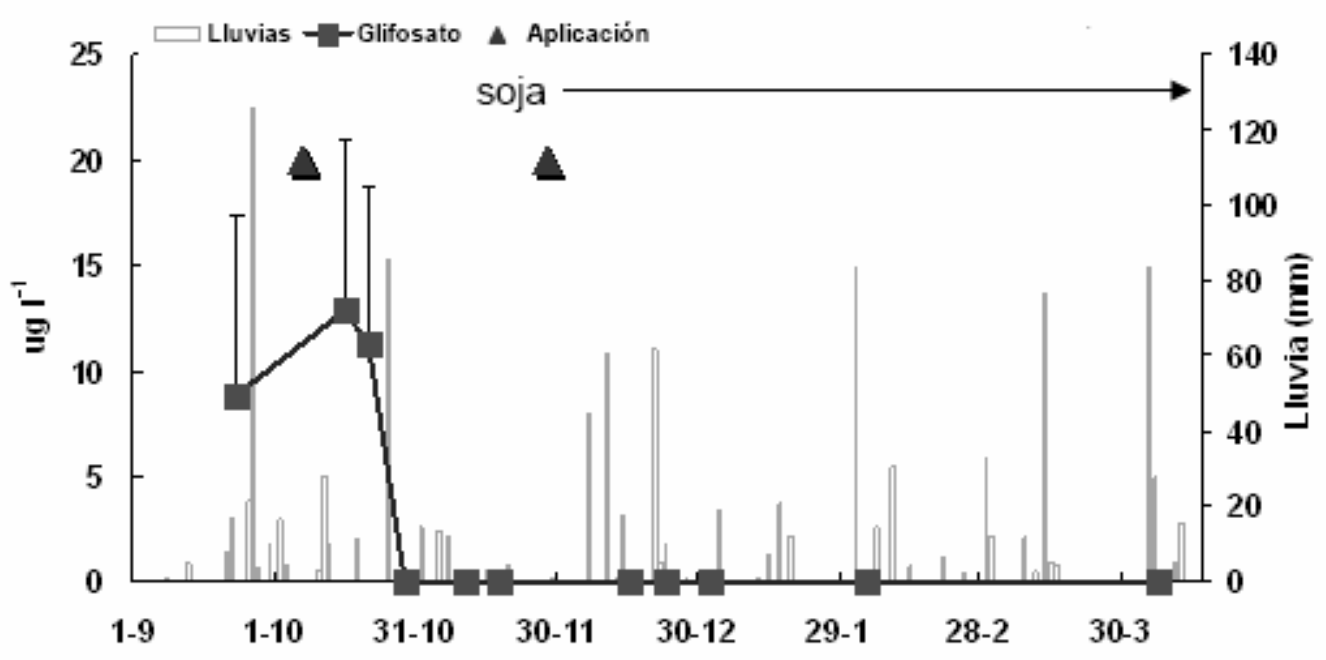

Fig. 3. Lluvias registradas en presiembra y durante el cultivo de soja (2002/2003) en Pergamino (barras) y concentración de glifosato en el agua de drenaje (línea). Los triángulos indican los momentos de aplicación de glifosato. La flecha superior indica el ciclo de la soja.

En Paraná el registro de lluvias durante la campaña 2006/2007 fue muy elevado con respecto al promedio anual histórico $(>50 \%)$. Desde la aplicación de glifosato en presiembra hasta finalizar el ciclo del cultivo llovieron $1430 \mathrm{~mm}$ y el $10 \%$ se perdió por escurrimiento. Se consideraron como efectivas (con potencialidad de producir escurrimiento) a las lluvias superiores a $12,5 \mathrm{~mm}$ (Wischmeier y Smith, 1958) que representaron el $88 \%$ de los eventos.

En tres fechas de muestreo se registraron concentraciones de glifosato en el agua escurrida en un rango de 1 a $12 \mathrm{\mu g} \mathrm{l}^{-1}$ (Figura 4). Las concentraciones de glifosato y AMPA del agua escurrida antes e inmediatamente después de la siembra fueron $6,47-8,27$ y $3,61-5,20 \mathrm{\mu g} \mathrm{I}^{-1}$, respectivamente. Un mes después de la siembra del cultivo las concentraciones registradas fueron bajas $(0,07-3,63$ y $0,36-0,87 \mathrm{\mu g} \mathrm{I}^{-1}$ para glifosato y AMPA, respectivamente). Dos y tres días después de la segunda y tercera aplicación del herbicida se registraron lluvias de 73 y $91 \mathrm{~mm}$, respectivamente, que produjeron escurrimiento que contenía glifosato. Screpanti et al (2005) detectaron picos de altas concentraciones de glifosato $\left(\sim 16 \mu \mathrm{g} \mathrm{I}^{-1}\right)$ en eventos de escurrimiento ocurridos 1 día después de la aplicación del herbicida. 
La concentración media de AMPA en el agua de escurrimiento fue similar a la de glifosato. Todos los muestreos registraron su presencia y hubo dos fechas de muestreo que presentaron concentraciones en un rango de 1 a $6 \mu \mathrm{g} \mathrm{l}^{-1}$, evidenciando la rápida velocidad de transformación del glifosato.

Las cantidades de glifosato y AMPA perdidas en el agua de escurrimiento durante el período de estudio, fueron muy bajas, menores al $0,05 \%$ de las cantidades aplicadas al cultivo de soja. Screpanti et al. (2005) también cuantificaron las pérdidas de glifosato con el agua de escurrimiento con respecto a las cantidades de ingrediente activo aplicadas y obtuvieron resultados muy inferiores a los nuestros (0.064\%o). En Entre Ríos, Díaz et al. (2008) analizaron muestras de agua superficial obtenidas en más de treinta represas para riego y no detectaron glifosato ni su metabolito AMPA.

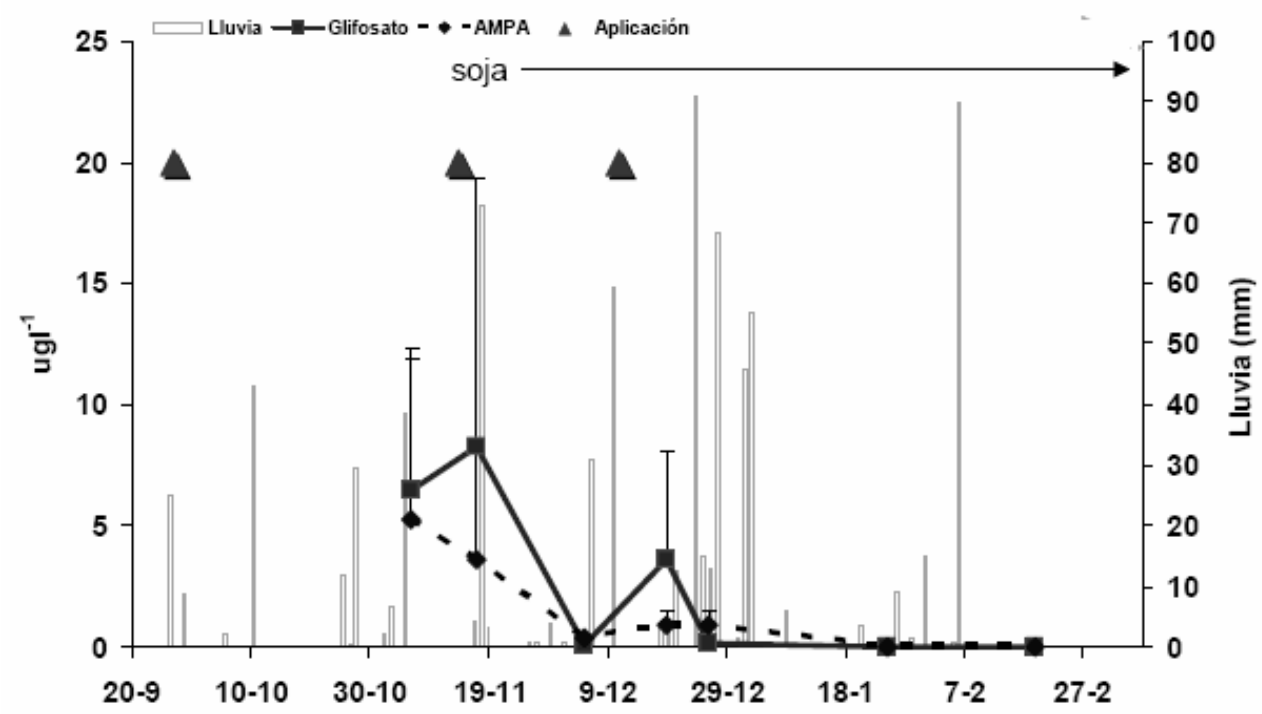

Fig. 4. Lluvias registradas en presiembra y durante el cultivo de soja (2006/2007) en Paraná (barras) y concentración de glifosato y AMPA en el agua de escurrimiento (líneas). Los triángulos indican los momentos de aplicación de glifosato. La flecha superior indica el ciclo de la soja.

La aplicación en post-emergencia del cultivo de soja en Pergamino se detectó con elevada variabilidad en la biomasa aérea (grano, vainas y tallos) presentando concentraciones inferiores al límite de $20 \mathrm{\mu g} \mathrm{g}^{-1}$ establecido por la USEPA (Tabla 1). La presencia de glifosato en la biomasa aérea representó $0,0005-0,001 \%$ de la cantidad aplicada en post-emergencia. Los valores hallados en grano, si bien son bajos, tienen importancia por los posibles procesos de bioacumulación de la cadena alimentaria (Lenardón et al., 2002).

Tabla 1. Concentración y cantidad de glifosato en grano, vaina y tallo de soja transgénica a la cosecha del cultivo en Pergamino.

\begin{tabular}{lcc}
\hline \multirow{2}{*}{$\begin{array}{l}\text { Parte de de } \\
\text { la planta }\end{array}$} & $\begin{array}{c}\text { Goncentración } \\
\left(\mu \mathrm{g} \mathrm{g}^{-1}\right)\end{array}$ & $\begin{array}{c}\text { Cantidad } \\
\left(\mu \mathrm{h} \mathrm{h}^{-1}\right)\end{array}$ \\
\hline Granos & $2,8 \pm 2,5$ & $10 \pm 9$ \\
\hline Vainas & $12,4 \pm 17,6$ & $86 \pm 45$ \\
\hline Tallos & $9,9 \pm 4,2$ & $49 \pm 23$ \\
\hline
\end{tabular}

El estudio del impacto ambiental que genera el uso de tecnologías agrícolas no es sencillo, ya que requiere de dispositivos experimentales adecuados que permitan el control de los insumos que se agregan al sistema y la correcta medición de los excedentes hídricos tanto en superficie como en profundidad. En todos los compartimentos analizados (tejidos vegetales, agua de escurrimiento y de drenaje) se registró la presencia de glifosato a pesar de la elevada variabilidad de las concentraciones. 


\section{CONCLUSIONES}

El estudio de la movilidad y transporte en el suelo de un herbicida ampliamente utilizado como el glifosato resulta de gran interés para el sector agrícola. Los resultados obtenidos sobre el aporte del cultivo de soja a la contaminación del agua por glifosato y AMPA mostraron su presencia en el agua de drenaje y de escurrimiento, en coincidencia con lluvias de primavera que favorecerían el pasaje rápido de agua hacia la capa freática o hacia cursos de agua superficial.

Si bien, las concentraciones encontradas fueron altas en relación a la norma europea, la cantidad de glifosato y AMPA que salió del sistema resultó muy pequeña, tanto por escurrimiento como por drenaje, representando menos del 0,03 y menos del $0,6 \%$ de las cantidades aplicadas, respectivamente. La aplicación de glifosato en postemergencia afectó además la calidad del grano.

Los máximos de alta concentración de glifosato y AMPA detectados en el escurrimiento y el drenaje luego de lluvias importantes y la intercepción del herbicida en función del momento del ciclo indican la relevancia de analizar aspectos relacionados al momento y condiciones de aplicación. La identificación del mejor momento para la aplicación de glifosato permitirá reducir el impacto sobre el sistema suelo-agua-planta.

\section{AGRADECIMIENTOS}

Este trabajo se realizó en el marco de los proyectos: PICT 2000 № 08054, PPR AEGA-221001 INTA y PE ERIOS-630021. Agradecemos la colaboración de Néstor Garciarena, José Oszust, Carlos Acosta, Alberto Rondán, Adolfo Sosa y Fernando Rimatori.

\section{REFERENCIAS}

Arregui, M.C., y otros cinco autores, Monitoring glyphosate residues in transgenic glyphosate resistant soybean, Pest Manag Sci.: 160(2),163-167 (2004)

Borggaard, O.K. y A.L. Gimsing, , Fate of glyphosate in soil and the possibility of leaching to ground and surface waters: a review. Pest Manag Sci 64(4): 441-456 (2008)

Byer, J.D., y otros cuatro autores, Low cost monitoring of glyphosate in surface waters using the ELISA method: an evaluation, Environ Sci Technol 42: (16) 6052-6057 (2008)

Díaz, E.L., y otros siete autores, Evaluación de residuos de plagicidas en suelos y aguas cultivados con arroz en Entre Ríos, Actas del XXI Congreso Argentino de la Ciencia del Suelo. San Luis, 13 al 16 de mayo (2008).

Elliott, J.A., Cesna, A.J., Best, K.B., Nicholaichuk, W. y L.C. Tollefson, Leaching rates and preferential flow of selected herbicides through tilled and untilled soil, J Environ Qual 29:1650-1656 (2000)

Flury, M., Experimental evidence of transport of pesticides through field soils-a review. J Environ Qual 25:25-45 (1996)

Hall, A.J., Rebella, C.M., Ghersa, C.M., y J.Ph. Culot, Field-crop systems of the pampas, In Field Crop Ecosystems. Ecosystems of the World by Pearson, C.J. (Ed.), pp. 413-450, Elsevier, Amsterdam (1992)

Jaynes, D.B., Ahmed, S.I., Kung K-J.S. y R.S. Kanwar, Temporal dynamics for preferential flow to a subsurface drain, Soil Sci Soc Am J 65:1368-1376 (2001)

Lenardón, A., y otros cinco autores, Plaguicidas en diversos medios: experiencias y resultados, Actas del II Taller de Contaminación por Agroquímicos, AIANBA, Pergamino, Buenos Aires, Argentina, 23 de agosto (2002)

Petersen, C.T., Holm, J., Koch, C.B., Jensen, H.E. y S. Hansen, Movement of pendimethalin, ioxynil and soil particles to field drainage tiles, Pest Manag Sci 59:85-96 (2002). 
Peruzzo P.J., Porta A.A. y A.E. Ronco, Levels of glyphosate in suface waters, sediments and soils associated with direct sowing soybean cultivation in north pampasic region of Argentina, Environ Pollut (en linea) www.sciencedirect.com (2008)

Portela S.I., Andriulo A.E., Jobbágy E.G. y M.C. Sasal, Water and nitrate exchange between cultivated ecosystems and groundwater in the Rolling Pampas, Agric. Ecosyst. Environ.: 134, $277-$ 286 (2009)

SAGPYA. Informe en línea http://www.sagpya.mecon.gov.ar/SAGPyA/agricultura/agua_y_suelos/ 05-fertilizantes/panorama.pdf Acceso: 21-09-2009. (2008)

Screpanti C. y C. Accinelli, Glyphosate and glufosinate-ammonium runoff from a corn-growing area in Italy, Agron Sustain Dev 25:3) 407-412 (2005)

Sprankle, P., Meggit, W.F. y D. Penner, Adsorption, mobility and microbial degradation of glyphosate in the soil, Weed Sci. 23: 229-234 (1975)

USEPA., Occurrence Estimation Methodology and Occurrence Findings for Six-Year Review of National Primary Drinking Water Regulations. Glyphosate - DRAFT, EPA Report/815-D-02-005, Office of Water, 192-197, USA (2002)

Vereecken H, Mobility and leaching of glyphosate: a review, Pest Manag Sci 61:139-1151 (2005).

Villholth, K.G., Jarvis, N.J., Jabobson, O.H. y H. de Jonge, Field investigations and modelling of particle-facilitated pesticide transport in macroporous soil. J Environ Qual 29:1298-1309 (2000)

Wischmeier W.H. y D.W. Smith, Rainfall energy and its relationship to soil loss, Trans. Am. Geo. Un.: 39, 285-291 (1958) 